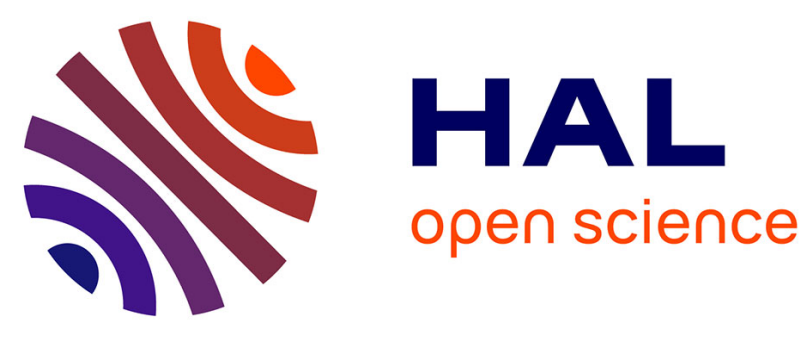

\title{
Cooperative Adaptive Cruise Control in Real Traffic Situations
}

\author{
Vicente Milanés, Steven E. Shladover, John Spring, Christopher Nowakowski, \\ Hiroshi Kawazoe, Masahide Nakamura
}

\section{- To cite this version:}

Vicente Milanés, Steven E. Shladover, John Spring, Christopher Nowakowski, Hiroshi Kawazoe, et al. Cooperative Adaptive Cruise Control in Real Traffic Situations. IEEE Transactions on Intelligent Transportation Systems, 2014, 15, pp.296 - 305. 10.1109/TITS.2013.2278494 • hal-01091154

\section{HAL Id: hal-01091154 https://hal.science/hal-01091154}

Submitted on 4 Dec 2014

HAL is a multi-disciplinary open access archive for the deposit and dissemination of scientific research documents, whether they are published or not. The documents may come from teaching and research institutions in France or abroad, or from public or private research centers.
L'archive ouverte pluridisciplinaire HAL, est destinée au dépôt et à la diffusion de documents scientifiques de niveau recherche, publiés ou non, émanant des établissements d'enseignement et de recherche français ou étrangers, des laboratoires publics ou privés. 


\title{
Cooperative Adaptive Cruise Control in Real Traffic Situations
}

\author{
Vicente Milanés, Steven E. Shladover, John Spring, Christopher Nowakowski, Hiroshi Kawazoe and \\ Masahide Nakamura
}

\begin{abstract}
Intelligent vehicle cooperation based on reliable communication systems contributes not only to reducing traffic accidents, but also to improving traffic flow. Adaptive Cruise Control (ACC) systems can gain enhanced performance by adding vehicle-vehicle wireless communication to provide additional information to augment range sensor data, leading to Cooperative ACC (CACC). This paper presents the design, development, implementation and testing of a CACC system. It consists of two controllers, one to manage the approaching maneuver to the leading vehicle and the other to regulate car-following once the vehicle joins the platoon. The system has been implemented on four production Infiniti M56s vehicles, and this paper details the results of experiments to validate the performance of the controller and its improvements with respect to the commercially available ACC system.
\end{abstract}

Index Terms-Cooperative Adaptive Cruise Control (CACC), adaptive cruise control (ACC), intelligent driving, cooperative vehicles, connected vehicles, intelligent transportation systems (ITS)

\section{INTRODUCTION}

Significant developments in Advanced Driver Assistance Systems (ADAS) have been achieved during the last decade. Intelligent systems based on on-board perception/detection devices have contributed to improve road safety [1]. The next step in the development of ADAS points toward vehicleto-vehicle $(\mathrm{V} 2 \mathrm{~V})$ communications to obtain more extensive and reliable information about vehicles in the surrounding area, representing a cooperative ITS system. Using wireless communication, potential risk situations can be detected earlier to help avoid crashes and more extensive information about other vehicles' motions can help improve vehicle control performance. Research projects have been conducted throughout the world to define the requirements for an appropriate vehicular communication system and its possible applications [2].

Although most of the V2V cooperative ITS applications have been focused on improving collision avoidance and safety [3], the extension of the commercially available Adaptive Cruise Control (ACC) system toward the Cooperative ACC (CACC) system has a high potential to improve traffic flow

V. Mílanés wants to especially thanks to the ME/Fulbright program and the Center for Automation and Robotics (CAR,UPM-CSIC) for its support in the development of this work.

V. Milanés, S. Shladover, J. Spring and C. Nowakowski are with the California PATH Program of the Institute of Transportation Studies, University of California, Richmond, CA 94804. (email: vicente.milanes@berkeley.edu, steve@path.berkeley.edu, jspring@path.berkeley.edu, chrisn@path.berkeley.edu)

H. Kawazoe and M. Nakamura are with the ITS Advanced and Product Engineering Group, Nissan Motor Co., Ltd. Kanagawa 243-0123 Japan. (email: kawazoe@mail.nissan.co.jp,n-masa@mail.nissan.co.jp) capacity and smoothness, reducing congestion on highways. By introducing $\mathrm{V} 2 \mathrm{~V}$ communications, the vehicle gets information not only from its preceding vehicle -as occurs in ACCbut also from the vehicles in front of the preceding one. Thanks to this preview information, oscillations due to speed changes by preceding vehicles can be drastically reduced. Benefits from including communications in ACC systems have been widely studied in recent years [4], [5], [6].

Prior experimental results using vehicle-vehicle cooperation to improve vehicle following performance were achieved by the California Partners for Advanced Transit and Highways (PATH) in 1997 [7], [8] when a platooning maneuver involving eight fully-automated cars was carried out using wireless communication among vehicles -mainly for longitudinal control- and magnetic markers in the infrastructure -mainly for lateral control. Based on the idea of a leading vehicle guiding several followers, the Safe Road Trains for the Environment (SARTRE) European Union project has developed virtual trains of vehicles in which a leading vehicle with a professional driver takes responsibility for each platoon [9]. That concept of the professional driver in the first vehicle was originally developed in the European project called CHAUFFEUR [10].

Specifically related to CACC implementations in production cars, two important projects were recently conducted in the Netherlands. The Connect \& Drive project, funded by the Dutch Ministry of Economic Affairs, carried out a CACC demo using six passenger vehicles [11] adopting a constant time gap spacing policy. For the Grand Cooperative Driving Challenge (GCDC) competition in 2011, nine heterogeneous vehicles from different European research institutions tried to perform a two-lane CACC platoon [12]. This competition revealed some of the most important problems to be solved before bringing this technology into production, including the communication systems reliability. From the control point of view, most of the implementations were based on proportional, proportional-derivative feedback/feedforward controllers [13], [14], [15] or Model Predictive Control (MPC) techniques [16], [17].

When it comes to designing a CACC system, string stability plays a key role [18]. The goal is designing a system able to reduce disturbances propagated from the leading vehicle to the rest of the vehicles in the platoon. There are two different approaches to car following gap regulation: one based on constant spacing or one based on constant time gap. A comparative study between them, where CACC stability was discussed, was presented in [19]. Several papers have dealt with string stability analysis and simulations [20], [21], [22], 
based on simplified theoretical models of ACC vehicle following behavior, and have shown encouraging results. However real production ACC systems have significant response delays that have not been represented in the prior theoretical analyses, but which destabilize the vehicle following responses. Consequently those theoretical analyses have produced unrealistically optimistic estimates of the traffic stability impacts of ACC.

In previous PATH research, a CACC involving two vehicles was tested with very favorable results [23], [24], [6]. Building on that previous work, this paper describes a new control system design and implementation that is integrated in four production vehicles. A constant time-gap car following strategy was implemented similar to the commercial ACC, but with the availability of significantly shorter time-gap settings. This is achievable because $\mathrm{V} 2 \mathrm{~V}$ communications permit tighter control of vehicle spacing and guarantee string stability, so that inter-vehicle time-gap settings significantly shorter than the production ACC time-gap settings are comfortable and acceptable to drivers [24]. The whole system was then tested in real traffic scenarios in order to compare its performance to the production ACC system installed in the vehicles. Cut-in and cut-out maneuvers were also tested to evaluate the controller under regular traffic circumstances, emulating everyday traffic situations.

The rest of the paper is organized as follows. Section II presents a brief explanation about the production vehicles used in the experimental phase, the control architecture implemented on each vehicle and the vehicle model. The control system that has been implemented based on a gap closing controller and a gap regulation controller is explained in Section III. Several experiments to validate the proposed systems are included in Section IV. Finally, some concluding remarks are given in Section V.

\section{EXPERIMENTAL VEHICLES}

Four production Infiniti M56s (see Fig. 1) were used as the experimental vehicles. These vehicles are rear-wheel drive with a 420-hp 5.6 liter V8 gasoline engine. They were factory equipped with lidar-based ACC, lane departure warning (LDW) and blind spot detection systems. Additionally, a 5.9 GHz Dedicated Short Range Communication (DSRC) system with a differential Global Positioning System (GPS) incorporated in a Wireless Safety Unit (WSU) was supplied by DENSO. Control was implemented through a dSpace MicroAutoBox which received data from both the WSU and the production vehicle's Controller Area Network (CAN). In particular, lidar data from the first and immediately preceding vehicles and data from on-board vehicle sensors -speed, acceleration, and yaw rate- were used by the control computer.

\section{A. Control architecture}

As previously stated, a factory installed ACC controller was available in the vehicles. This controller sends target speed commands to the vehicle's actuators. The same control variable is available for the CACC system, but it needs to act through the commercial system that controls the throttle

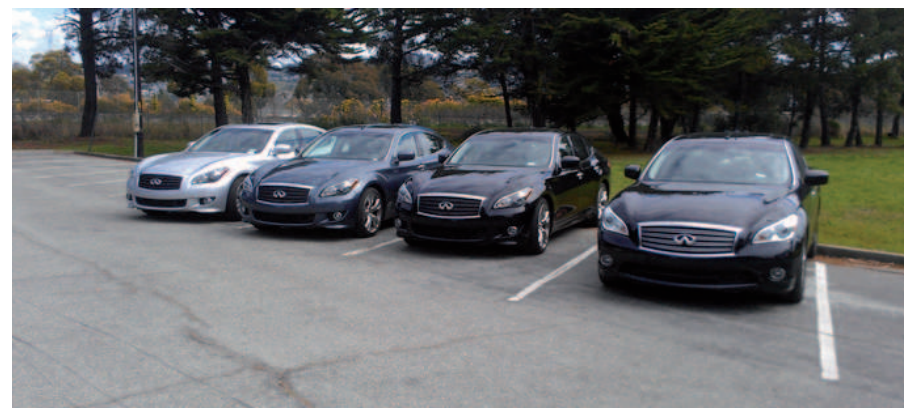

Fig. 1. Experimental M56s vehicles

and brake pedals, i.e. the low-level controller. This constraint somewhat limits the options when it comes to designing the controller since the low-level controller could not be modified, but it still provides adequate dynamic range for controlling the vehicle under non-emergency transient conditions. Figure 2 shows the block diagram for the vehicle control architecture. It consists of a classical robotics control architecture divided into three stages:

- Perception phase where all information from the sensors installed in the vehicle is received. Two sources can be distinguished. On one hand, information coming from the WSU system, where all data communicated by other vehicles in the platoon -speed, acceleration, distance to preceding vehicle, current time gap, control activation and so on- is received and included on the CAN bus data structure. This data also includes detection and assignment of the vehicle position sequence in the platoon, which is carried out by the WSU using its GPS with Wider Area Augmentation System (WAAS) differential corrections. On the other hand, information is obtained from the on-board sensors, such as factory available lidar measurements (relative distance to the preceding vehicle), odometer (current speed) and acceleration, and flag signals (to get information about the interaction of the driver with the driver interface such as activation or deactivation of the system or gap setting selection). The driver interface buttons, located on the right side of the steering wheel, are also used for the CACC controller.

- Planning stage includes the high-level controller. Both controllers, the commercial ACC system and the newly developed CACC system, are available during the tests so the driver can switch between them in real time. For switching, the control code reads the CAN bus information coming from the transmission mode selection button-i.e., eco-driving mode, sport mode, standard mode or snow mode. When the sport mode is chosen, the highlevel controller will take the CACC controller output. When any of the other modes is chosen, the production ACC controller output will be sent to the low-level controller. The CACC controller code has been developed in Matlab/Simulink and is loaded in the vehicle using a dSpace MicroAutoBox which is connected to the vehicle via the CAN bus, where the target speed commands are sent. The CACC controller can be deactivated in the 


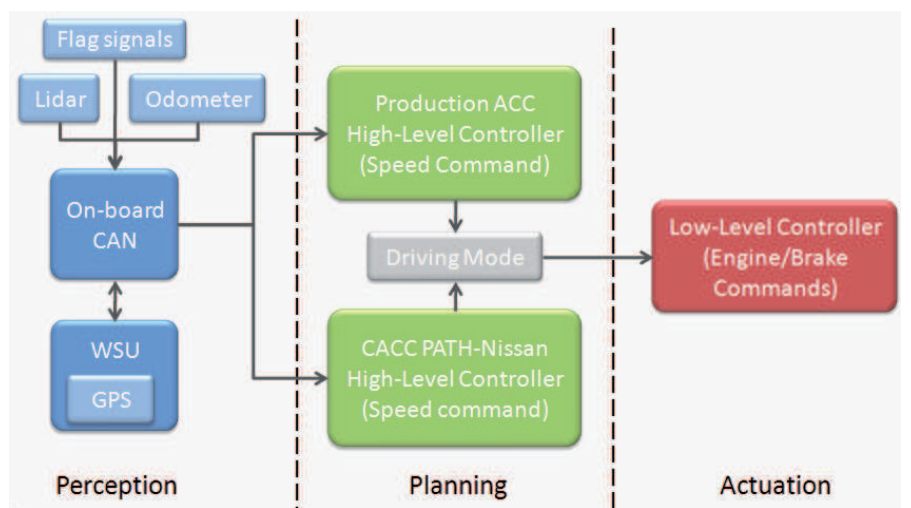

Fig. 2. Control architecture block diagram

same way as the commercial ACC, either using the driver interface buttons or pressing the brake pedal.

- Actuation phase is in charge of executing target reference commands coming from the planning stage. As previously stated, this low-level controller is in charge of converting the target speed commands into throttle and brake actions, using the factory ACC controller.

\section{B. Vehicle model}

The vehicle dynamic model, to be used for evaluating vehicle performance from the string stability point of view, is identified based on its step responses to different speed changes. For these tests, a simple open-loop controller is in charge of generating speed target commands to the vehicle. Once the vehicle is stably driving at its current target speed, a new speed command for an accelerating or braking maneuver is sent to the vehicle. The Infiniti M56s vehicles are equipped with a powerful engine that produces fast and strong responses to changes in the target speed command. Considering this, a second order response model can be extracted from the experimental test data, where two different dynamic responses are clearly evident in the behavior of the vehicle during the accelerating and braking phases. Overshoot occurs on the braking response since a high engine braking force - especially in the sport driving mode, where the CACC controller was designed- is added to the friction braking action. Two secondorder models with time delay were identified from the test data, with the following structure

$$
F(s)=\frac{k}{s^{2}+2 \theta \omega_{n} s+\omega_{n}^{2}} e^{-T_{d} s}
$$

with $k, \theta, \omega_{n}$ and $T_{d}$ being the static gain, damping factor, natural frequency and time delay respectively. Parameters for both models -i.e. braking and acceleration responsesare defined in Table I. They are obtained using the Matlab System Identification Tool, which permits selection among different candidate transfer functions. This transfer function for representing vehicle behavior was chosen as a trade-off between simplicity (second order model) and goodness of fit (over 95\%). Responses for both models to a speed change are depicted in Fig. 3. One can appreciate how well the model fits the response of the real vehicle. This model incorporates
TABLE I

ACCELERATING AND BRAKING MODEL PARAMETERS

\begin{tabular}{ccccc} 
& $k$ & $\theta$ & $\omega_{n}$ & $T_{d}$ \\
\hline \hline Accelerating & 0.156 & 0.661 & 0.396 & 0.146 \\
Braking & 1.136 & 0.5 & 1.067 & 0.287 \\
\hline
\end{tabular}
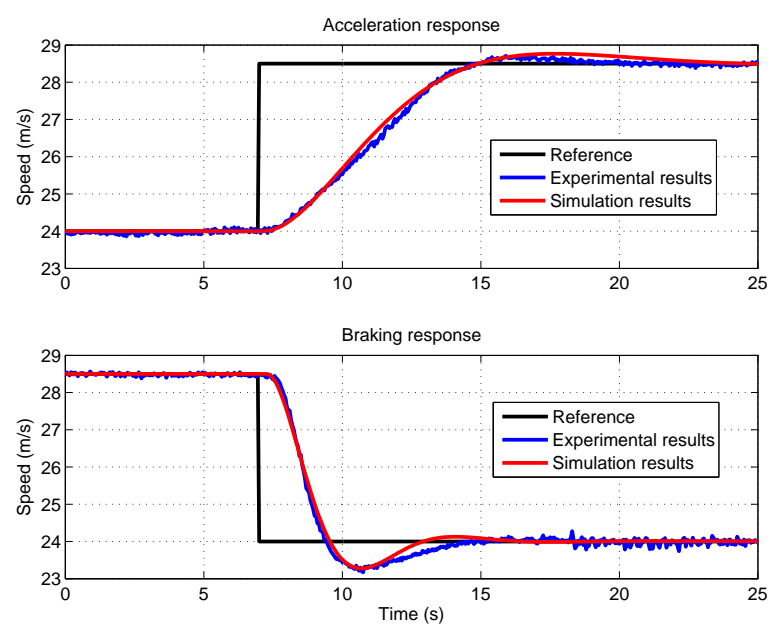

Fig. 3. Vehicle's longitudinal dynamics response for acceleration and braking maneuvers

both the dynamics of the vehicle and the low-level controller in charge of managing throttle and brake actions.

\section{CONTROL DESIGN}

The goal of the CACC controller is maintaining the driverdesired time gap to the preceding vehicle in any traffic circumstance, with both smoothness and accuracy. The ACC driver interface is used to manage the CACC controller. It includes buttons for activating and deactivating the ACC controller, three gap settings and the option of setting, increasing or decreasing the cruise control speed, in case no vehicle is detected in front of the ego-vehicle. For the ACC factory system, the available gap settings are 1.1, 1.6 and 2.2 seconds. For the CACC system, the shortest gap is set at 0.6 seconds. This value has been chosen based on estimates of the separation needed to enable crash avoidance under emergency conditions, as well as previous tests of acceptance by drivers from the general public [24]. The other two available CACC gap settings are 0.9 and 1.1 seconds. There were two limitations when designing the CACC controller: it is not possible to access or modify the low-level controller; and acceleration and deceleration are limited to maximum values ( $0.1 g$ and $0.28 g$ respectively) by the low-level controller.

The controller has been divided in two stages. The first stage occurs when the CACC system is activated and there is no vehicle in front of it or the ego-vehicle is far away from the preceding one. In these cases, the vehicle is usually following its set speed so an approaching maneuver has to be carried out before switching to a car-following policy. This controller -the gap closing controller- has to be as smooth as possible to permit a good transition to the gap regulation controller. It is also used in case of cut-out maneuvers when a vehicle in 


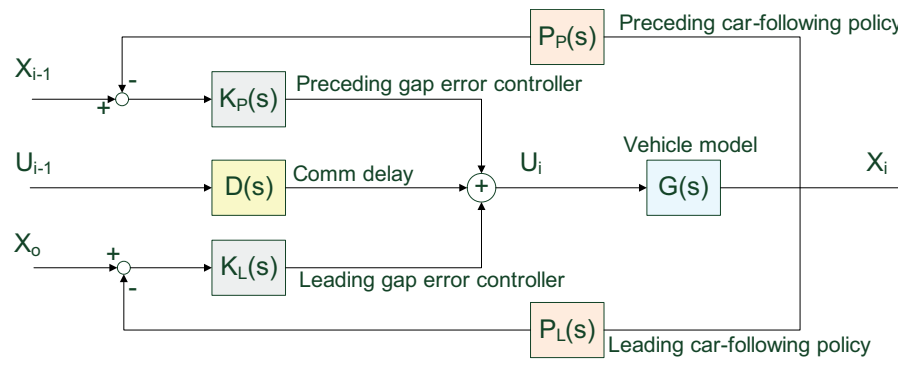

Fig. 4. CACC control structure block diagram

the middle of the platoon decides to leave and the following one has to cover the cut-out vehicle gap.

Once the vehicle has joined its predecessor, the second stage controller -the CACC gap regulation controller- is in charge of implementing the car-following policy depending on the time gap selected by the driver. Three different time gaps are available, following the production ACC structure. This controller is also in charge of managing cut-in maneuvers when a non-equipped vehicle merges into the platoon. Both controllers have been designed to be consistent with how a human driver handles each driving situation.

\section{A. Gap regulation controller}

Once the vehicle is close enough and the gap closing maneuver has finished, the system switches to the gap regulation controller, which is the core of the CACC control system. Commercially available ACC systems try to reduce the gap error $\left(x_{e}\right)$ between the ego-vehicle and the preceding one. Information from the lidar/radar is used to reduce gap error $\left(x_{e}\right)$ between desired time gap $\left(t_{g}\right)$ and relative distance $\left(x_{r}=x_{p}-x_{f}\right)$-where $x_{p}$ is the current position of the preceding vehicle and $x_{f}$ is the position of the ego-vehicle, following the next expression

$$
x_{e}=x_{r}-v_{f} t_{g}
$$

where $v_{f}$ is the speed of the ego-vehicle. For this purpose, a controller, $K_{P}(s)$ based on a standard PD-control structure [25], [26], can be easily adjusted to get stability with respect to the immediately preceding vehicle but, as previously demonstrated [27], this is not enough to guarantee string stability.

Exchanging information among vehicles using wireless communications permits improving the vehicle's response as well as significantly reducing time gaps, keeping safety. When it comes to designing a CACC system, string stability is one of the main goals. It can be defined as the system's ability to reduce perturbations downstream, avoiding that leading vehicle speed changes cause amplification in the rest of the vehicles [28]. According to [29], it can be defined as

$$
|S S(s)|=\left|\frac{X_{i}(s)}{X_{i-1}(s)}\right| \leq 1, i \geq 2
$$

where $i$ indicates the position of the vehicle in the platoon.

Figure 4 shows the CACC controller block diagram where $G(s)$ represents the vehicle model; terms $P_{P}(s)$ and $P_{L}(s)$ correspond to the car-following policy with respect to the preceding and the leading vehicle respectively; terms $K_{P}(s)$ and $K_{L}(s)$ represent time-gap error regulation controller for the preceding and the leading vehicle respectively; $U_{i}$ and $U_{i-1}$ correspond to the control action for the ego-vehicle and the preceding vehicle (coming from the wireless communication system) respectively; the term $D(s)=e^{-\delta s}$ represents the time delay in the wireless communication; and $X_{i}, X_{i-1}$ and $X_{0}$ represent the positions of the ego-vehicle, the preceding and the leading vehicle position in the platoon. The controller is formed by three main terms: one of them is in charge of keeping the current speed but, instead of using the ego-vehicle or preceding vehicle speed [23], the preceding vehicle target speed $\left(U_{i-1}\right)$ is used as a feedforward term. This permits improving the vehicle response time to speed changes and reducing delays in the transition between throttle and brake actuations. The other two terms try to keep the errors with respect to the preceding vehicle $K_{P}(s)$ and the leading vehicle $K_{L}(s)$ as small as possible. Both terms correspond to a classic PD structure defined as

$$
\begin{aligned}
& K_{P}(s)=k_{1} s+k_{2} \\
& K_{L}(s)=k_{3} s+k_{4}
\end{aligned}
$$

where car-following policies can be defined as

$$
\begin{aligned}
& P_{P}(s)=h_{P} s+1 \\
& P_{L}(s)=h_{L} s+1
\end{aligned}
$$

with $h_{P}$ and $h_{L}$ being the time-gap target values with respect to the preceding and leading vehicles respectively.

As previously stated, the vehicle clearly exhibits different dynamics in the acceleration and braking phases that can be modeled by second-order functions $s G(s)=F(s)$ so the position $X_{i}(s)$ for a vehicle in the platoon can be determined as

$$
X_{i}(s)=G(s) U_{i}(s)
$$

where $U_{i}(s)$ is the target speed command for that vehicle. Assuming the vehicles start from rest and using equation (8), the relation between the ego-vehicle and the preceding one is given by

$$
X_{i}(s)=\frac{D(s)+G(s) K_{P}(s)}{1+G(s)\left[K_{P}(s) P_{P}(s)+K_{L}(s) P_{L}(s)\right]} X_{i-1}(s)
$$

Following the criteria defined for string stability, the goal is to keep the Bode magnitude below the unity gain of both vehicle dynamics, i.e. acceleration and braking responses. Control gains were firstly modified using tuning tools from Matlab/Simulink to fulfill the requirements. Final tuning was carried out in the experimental vehicle in order to not only get an accurate response from the car-following policy point of view but also a smooth riding quality from the driver perspective. Table II shows the final parameters selected for the preceding and leader car-following policy controllers. The 
TABLE II

$K_{P}$ AND $K_{L}$ CONTROLLER PARAMETERS

\begin{tabular}{cccc}
$k_{1}$ & $k_{2}$ & $k_{3}$ & $k_{4}$ \\
\hline \hline 0.45 & 0.25 & 0.15 & 0.1
\end{tabular}

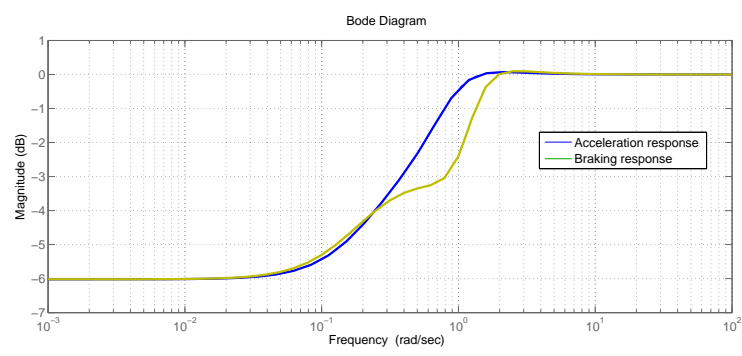

Fig. 5. String stability frequency analysis

same parameters were used for accelerating and braking maneuvers since good responses were obtained for both dynamics and they fulfilled string stability criteria. Control gains of the controller in charge of minimizing the error with respect to the leading vehicle are smaller than the ones in the controller that tries to reduce the error with respect to the preceding one. This is consistent with the need to ensure safety (avoiding potential impact with preceding vehicle) and to reduce the perceived variability of the gap relative to the preceding vehicle to promote driver confidence in the system.

Figure 5 shows responses for both dynamics -i.e. acceleration and braking- in the frequency domain, showing that the string stability criterion is fulfilled. The difference in both dynamics is also reflected in the frequency analysis. For the controller design, it was assumed that there is no delay in the wireless communication system, i.e. $D(S)=1$. This assumption was subsequently confirmed during the experiments, when the packet loss percentage was insignificant. Effects of packet loss on string stability are out of the scope of this paper.

\section{B. Gap closing controller}

For approaching the preceding vehicle, a simple linear function depending on the relative speed and distance between vehicles and the desired deceleration has been used. It can be configured according to the driver's preference. Fig. 6 shows the gap closing controller operation where $d r_{\text {start }}$ represents the initial inter-vehicle distance when a preceding vehicle is detected; $v r_{\text {start }}$ indicates relative speed between vehicles; $d r_{\text {end }}$ represents the distance when the controller switches to the gap regulation mode; and $v r_{\text {end }}$ indicate the final relative speed equal to zero. The ego-vehicle braking maneuver depends on the desired deceleration-i.e. $a_{\text {smooth }}, a_{\text {medium }}$ or $a_{\text {hard }}$ in the graphic. This parameter can be configured to match the driver's preferences. The smoother the deceleration, the earlier the vehicle starts to brake. The controller has been tested both following a set speed before joining a platoon and in a cut-out maneuver when the vehicle initially switched from the gap regulation controller to the gap closing controller, and then switched again to the gap regulation controller. Since this second option involves more complex situations, results of an

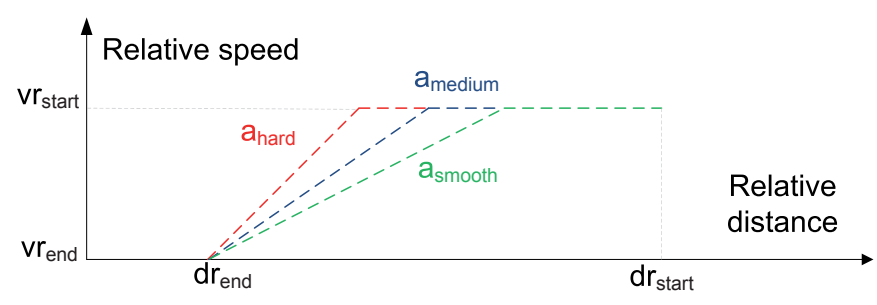

Fig. 6. Gap closing controller operation
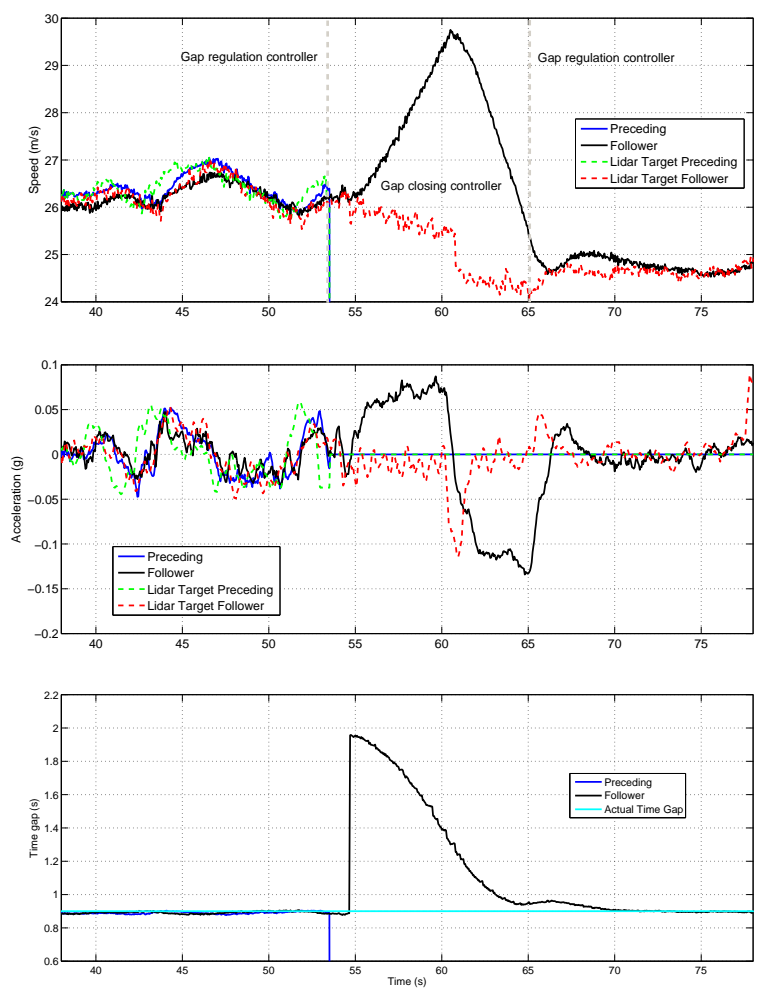

Fig. 7. Preceding car approaching maneuver in cut-out scenario

experiment for this situation are included to show the behavior of the gap closing controller.

Figure 7 depicts the performance of the gap closing controller during a three-vehicle CACC test. The top graph plots the evolution of the speeds of the vehicles and their forward vehicle speeds estimated from the lidar range measurements. The middle graph plots the vehicles' accelerations during the experiment. The bottom graph represents the time gaps between vehicles during the test. For notation, the leading vehicle is the first vehicle during the whole test; the preceding vehicle-solid blue line-is the one that starts the platoon in the second position and then cuts out; and, the follower vehiclesolid black line-is the one that begins in the third position and then performs the approaching maneuver. The preceding and follower vehicles are driving under CACC control with a time gap of 0.9 seconds and a set speed of $31.1 \mathrm{~m} / \mathrm{s}$. Forward vehicle speeds estimated from the lidar range measurements of the preceding vehicle-dotted green line-and the followerdotted red line-are also shown.

During the first 54 seconds, preceding and follower vehicles 
are driving in CACC following the leading vehicle in the platoon. One can appreciate how the speed estimated from the lidar range measurements of the follower-dotted red lineis tracking the preceding vehicle's speed-solid blue line. Close to second 54, the preceding vehicle changes lane (cuts out) so both its speed and lidar measurement have been removed for the sake of clarity. One can appreciate how the follower detects that its preceding vehicle has cut out and a sharp change in the time gap occurs. At that point, the system switches between the gap regulation controller and the gap closing controller. The follower starts to smoothly accelerate in order to close the gap with the leading vehicle. Considering the deceleration parameter constraint for this test $-0.1 \mathrm{~g}$, the vehicle starts to brake smoothly to create a new two-vehicle platoon with the leading vehicle. The new target vehicle is driven by a human driver so some speed changes-as occurs in real traffic drivingduring the gap closing maneuver are seen. It can be observed how the vehicle switches again to the gap regulation controller close to second 65 , where a change in the deceleration is observed. Then, the vehicle finishes the gap closing maneuver, perfectly fitting the desired time gap.

\section{EXPERIMENTAL RESULTS}

The CACC system was implemented in four Infiniti M56s vehicles equipped with $5.9 \mathrm{GHz}$ DSRC for wireless communication. Several trials were carried out with the vehicles to validate the proposed approach and the designed controller. In particular, three different test results are shown here. The first experiment consists of evaluating behavior of the carfollowing policy while the driver is changing the gap settings. The second experiment shows a realistic situation on highways when the ego-vehicle is following a leader, and another vehicle wants to take the next exit, executing both a cut-in and a cut-out maneuver in a relatively short period of time. The third experiment compares the CACC performance of the four vehicles following a moderate, but realistic, speed change profile with the performance of the production ACC system under the same conditions.

\section{A. Gap setting changes test}

For testing controller behavior when the driver chooses to change the gap setting, only two vehicles were used, one of them acting as the leading vehicle and the other one running the CACC controller. Figure 8 shows this test result. The upper plot shows the speed of both vehicles during the test; the middle plot depicts the acceleration; and the gap setting chosen by the driver is shown in the bottom graph, compared with the actual gap.

During the test, the leader accelerates and brakes slightly, emulating real traffic behavior. The experiment starts with a gap of 1.1 seconds, the longest CACC gap available on the driver interface. Around second 58, the driver changes to the middle gap setting -i.e. 0.9 seconds. The follower accelerates smoothly to reduce the gap. One can appreciate this transition in the time gap plot where no overshoot or sharp change occurs. The vehicle exhibits a similar behavior when it goes from the middle to the shortest gap -0.6 seconds. Then, the
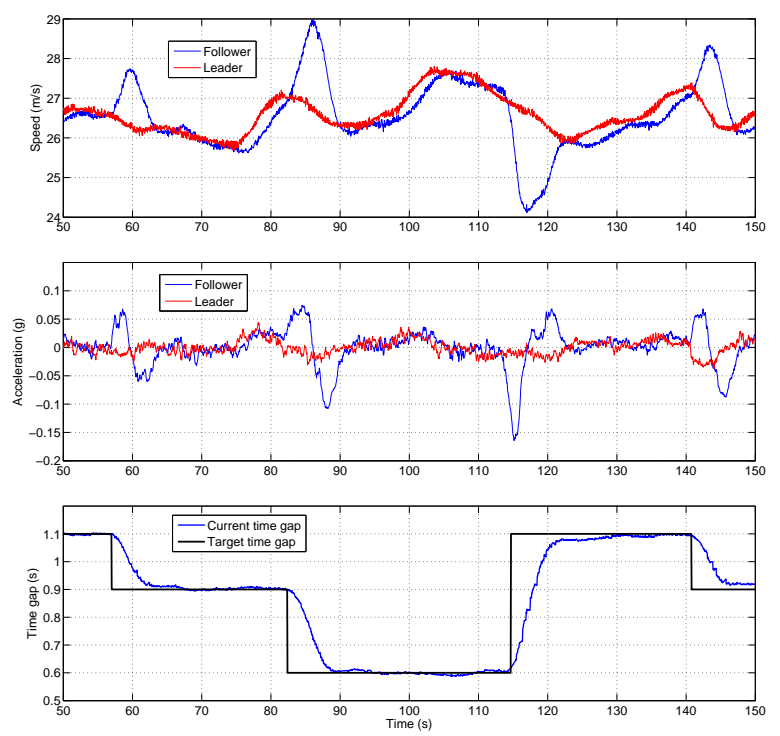

Fig. 8. Gap setting changes response

vehicle comes back to the longest gap. In this case, it is driving at 0.6 seconds behind the leading vehicle when the reference gap is 1.1 seconds so a significant deceleration is observed on the part of the follower in order to achieve the desired gap. One can appreciate how time gap transitions among different gap settings are smooth, and once the desired gap has been reached, the car-following policy is well-maintained. Finally, around second 100, it can be noticed that there appears to be some lag in the speed response of the follower as the leader accelerates. Although this might intuitively appear as a delay in the response, the lag is actually an artifact of using a constant time-gap car-following policy, instead of a constantspacing car-following policy. As speed increases, so does the desired inter-vehicle distance. Examining the associated timegap plot, one can see that the time gap remained constant during the lead vehicle acceleration.

\section{B. Cut-in and cut-out test}

A regular situation, if the $\mathrm{CACC}$ platoon is driving in the right-most lane on a highway, is that sudden and unexpected cut-ins occur when other vehicles want to enter or leave the highway. In these situations, a vehicle carries out both a cutin and a cut-out maneuver in a short period of time. In this experiment, the system response to those situations is shown.

For the sake of clarity, a two-vehicle CACC platoon response is shown in this test, but the test has been performed successfully for a four-vehicle CACC platoon. For safety reasons, the chosen gap setting is 1.1 seconds. Figure 9 shows vehicle behavior during this experiment. As in the previous one, the upper graph shows vehicle speeds; the middle graph shows vehicle accelerations; and the bottom graph depicts the current and reference (desired) time gaps.

At the beginning, the leader is driving at a constant speed and the follower is perfectly tracking the leader's speed at the desired gap. Around second 205, a cut-in vehicle merges between them. This vehicle is detected by the lidar, as revealed 

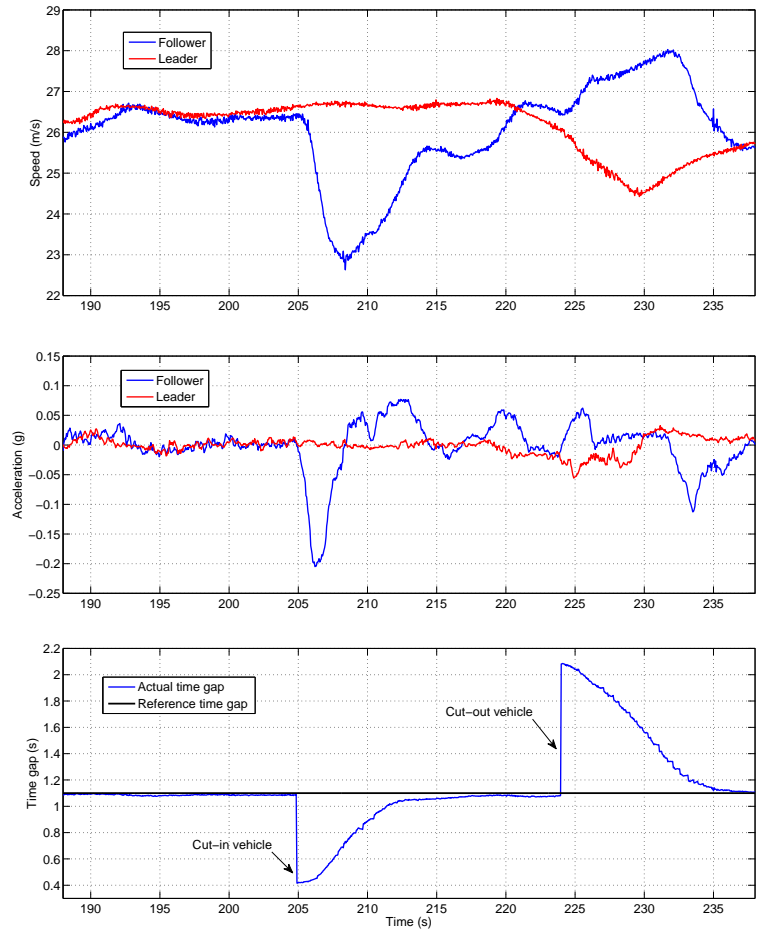

Fig. 9. Cut-in and cut-out vehicle response with CACC control

by the sudden change in the time gap, whose value decreases to 0.42 seconds. The follower brakes in order to increase the gap to the new vehicle and, after a few seconds, it reaches the desired gap. Then, around second 224, the cut-in vehicle cuts out by doing another lane change. Again, that movement is detected by the lidar and a sudden increase in the time gap occurs. The follower accelerates smoothly to achieve the desired gap with the original leader. One can notice the smooth transition toward the desired time gap in both the cutin and cut-out maneuvers without any abrupt behaviors. The hardest deceleration occurs when the vehicle cuts in, but this is because the time gap is drastically reduced, creating a potential safety hazard that needs to be reduced as quickly as possible. It is also important to note that deceleration is always within the comfort range, set at $\pm 0.2 g$ [30].

\section{Four vehicle test}

The last experiment shows the comparison between the production ACC system and the newly developed CACC controller. To this end, an automatic speed profile was designed and implemented on the leader car. It consisted of the following series of speed changes. In the first cycle, the vehicle drives at $25.5 \mathrm{~m} / \mathrm{s}$ for 10 seconds; then, it accelerates at a constant acceleration of $(1 / 80) \mathrm{g}$ to reach $29.5 \mathrm{~m} / \mathrm{s}$ speed. The speed remains constant for 10 seconds, before the vehicle starts to decelerate at a constant rate of $(1 / 80) g$ to come back to a constant speed of $25.5 \mathrm{~m} / \mathrm{s}$ for 10 seconds. The second cycle repeats the same acceleration and deceleration curve with a constant acceleration of $(1 / 40) \mathrm{g}$ and 15 seconds of driving at a constant speed at the top and bottom of each acceleration or deceleration event. The third and fourth cycles repeat the same pattern at $(1 / 20) g$ and $(1 / 10) g$ with 20 seconds of driving at a constant speed between acceleration and deceleration events. This profile emulates a real driving situation in moderately congested traffic, where repeated accelerations and decelerations happen. The speed variations have been constrained for this test so that it could be conducted safely on a public highway, mixed with other traffic.

Figure 10 shows the ACC system response to this profile, with the gap between vehicles set to 1.1 seconds, the shortest available on the production ACC. Before starting the profile, all vehicles drove with the ACC system activated and in a stable state. The leading vehicle drove as close as possible to the initial speed, $25.5 \mathrm{~m} / \mathrm{s}$, and then activated the profile.

Upon acceleration, note the oscillations, mainly on the third and fourth cars in the platoon, in both the vehicle speed and time gap, before stabilizing around second 30 at a constant time gap. In the subsequent acceleration cycles, note that the third and fourth car response delays are considerably larger and the overshoots in the speed during the last two accelerations caused an amplification downstream along the platoon. A considerable delay, close to 15 seconds, can also be noted in the response of the last car of the platoon. Finally, from the fourth vehicle driver's perception, his vehicle does not appear to be following any gap control policy because of the unstable behavior during the last speed change.

It is worth noting that this ACC system is not a degradation of our CACC controller from removing the feedforward but is the commercially available ACC that has been working in production cars with really good acceptability on the part of the drivers. It was designed with serious consideration of string stability, unlike many other ACC systems, and is actually more stable than some other commercially available systems. However, even its stability enhancing design features are not able to overcome the challenges associated with the lack of information available about the motions of the vehicles ahead of the immediately preceding vehicle

The same profile was tested for the CACC controller, and Figure 11 shows the results using the same time scale as for the ACC test results. For this test, the shortest gap -0.6 seconds for CACC- was used. One can appreciate how the overshoot of the third and fourth vehicles during the last two speed changes was eliminated. Additionally, there is no appreciable delay in the vehicles' responses, and the car-following policy is perfectly tracked by all vehicles in the platoon. These results clearly show how CACC control improves on ACC results for vehicle following and there has the potential to significantly improve traffic efficiency and safety.

Figures 12 and 13 detail the final braking transient when the leading car braking rate equals $0.1 \mathrm{~g}$. On the ACC graphic, the response delay is clearly seen since the last vehicle in the platoon -solid blue line- is still accelerating during the period in which the leading vehicle is braking. Both the significant delay and the amplification are observed in the acceleration graph. Most striking is the fact that the leading vehicle braking, $0.1 \mathrm{~g}$, is amplified by the last car to $0.3 \mathrm{~g}$, causing an uncomfortable behavior from the driver and passenger point of view. In contrast, the CACC graphic shows how the leading vehicle braking is attenuated by the last car, and the 

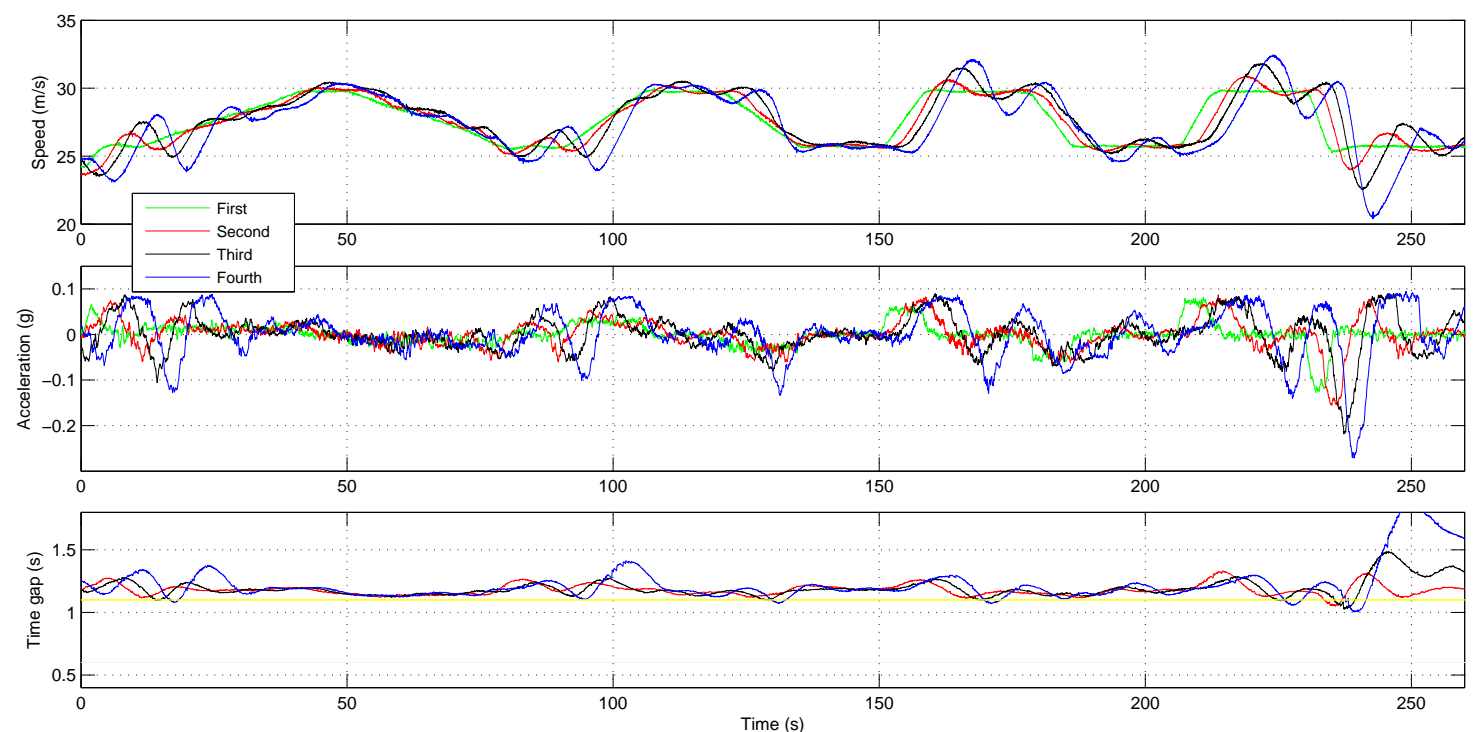

Fig. 10. Four vehicle ACC test results with speed profile in the leading vehicle
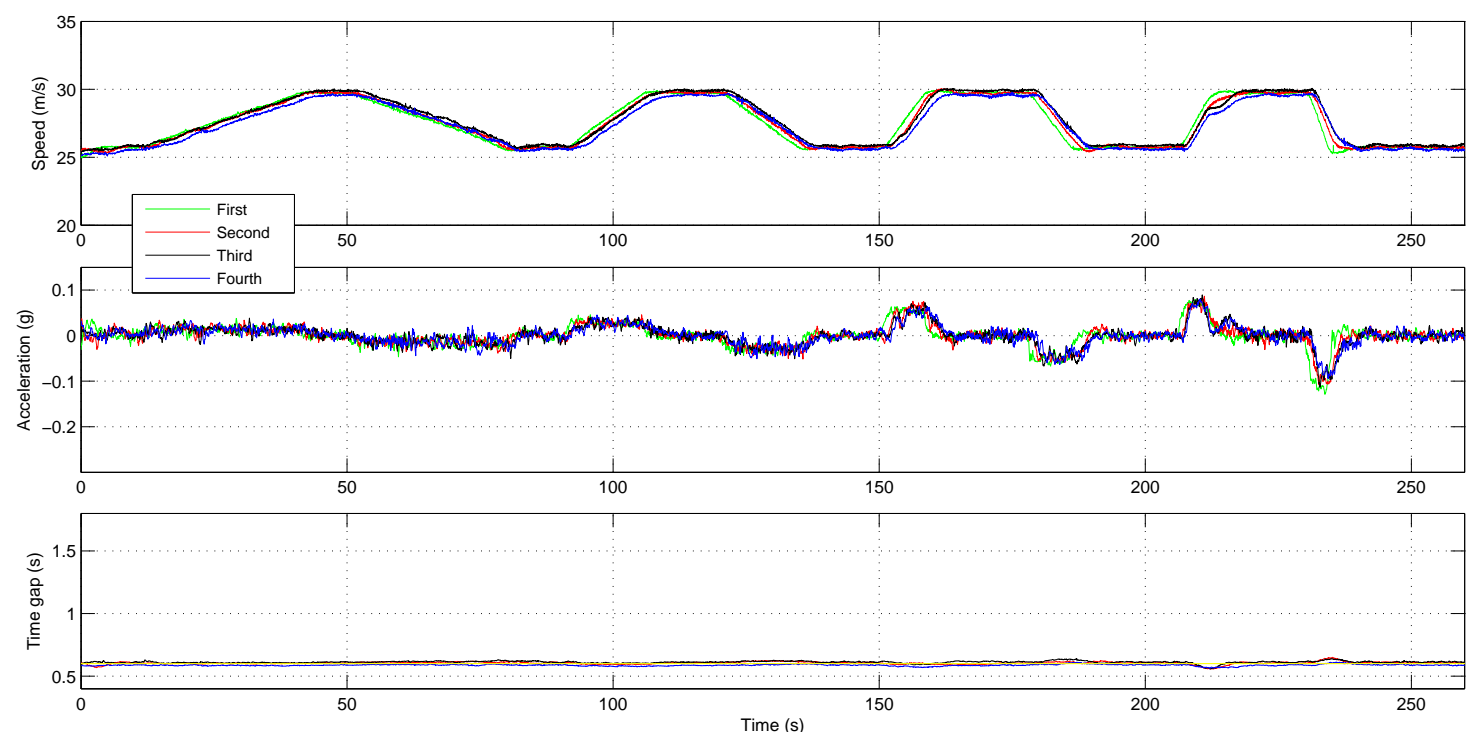

Fig. 11. Four vehicle CACC test results with speed profile in the leading vehicle

deceleration is actually lower than the initial $0.1 \mathrm{~g}$ disturbance during the event. The accurate tracking of the time gap policy for the hardest braking during the speed profile is also worth noting.

\section{CONCLUSION}

This paper presents the design, development, implementation and testing of an enhancement to commercially available ACC systems, based on introducing vehicle-to-vehicle communications, to produce CACC. The system has been implemented in four production Infiniti M56s vehicles equipped with DSRC devices for information exchange among vehicles.
The CACC controller design takes advantage of wireless communication information, introducing feedforward terms in the control logic, to enable significant reductions in intervehicular gaps. The system has been tested on public roads showing good performance. First, reduced gap variability was demonstrated. Then, the ability to gracefully handle unequipped vehicles cutting in and out was also validated. Finally, a comparative study between the production ACC system performance and the new CACC controller was carried out. The CACC clearly showed improvements in response time and string stability, indicating the potential for a CACC system to attenuate disturbances and improve highway capacity and 


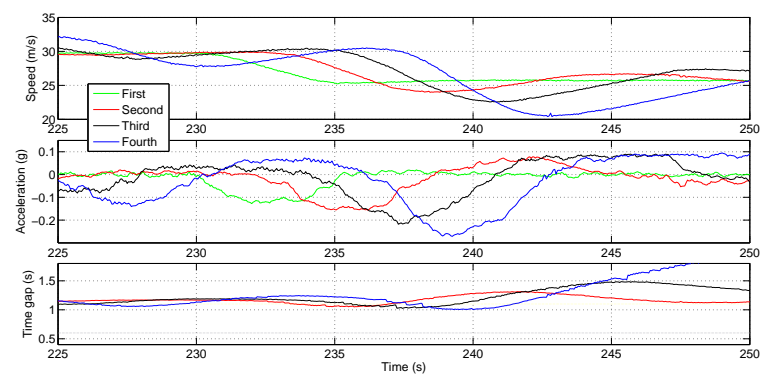

Fig. 12. Detail of the last deceleration for the ACC test using the speed profile

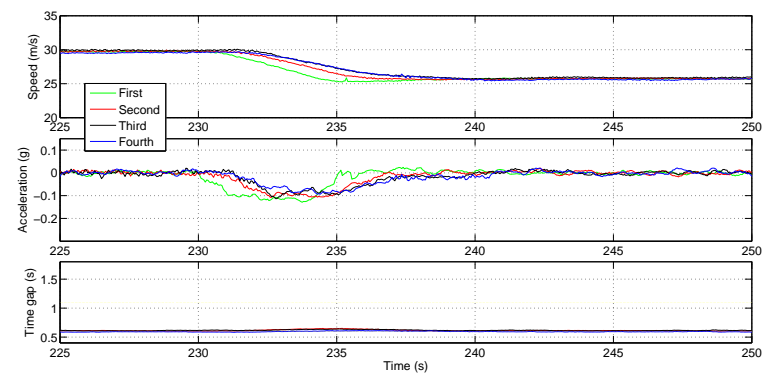

Fig. 13. Detail of the last deceleration for the CACC test using the speed profile

traffic flow stability. On-going and future research on this topic is mainly focused on assessing the potential magnitude of improvements that a CACC system might have on traffic response, both with respect to ACC and as a function of the market penetration.

\section{ACKNOWLEDGMENT}

The authors would like to thank Hiroshi Tsuda from Nissan Technical Center North America and Junichiro Funabashi from DENSO Corporation for the valuable discussions and for their support on vehicle testing. The authors would also like to thank Dave Nelson, Thang Lian, Somak Datta Gupta and Jing-Quan $\mathrm{Li}$ of California PATH for their support on vehicle testing. The PATH work on this project was sponsored by Nissan Motor Co. Ltd.

\section{REFERENCES}

[1] L. Li, D. Wen, N.-N. Zheng, and L.-C. Shen, "Cognitive Cars: A New Frontier for ADAS Research," IEEE Transactions on Intelligent Transportation Systems, vol. 13, no. 1, pp. 395-407, 2012.

[2] G. Karagiannis, O. Altintas, E. Ekici, G. Heijenk, B. Jarupan, K. Lin, and T. Weil, "Vehicular networking: A survey and tutorial on requirements, architectures, challenges, standards and solutions," IEEE Communications Surveys \& Tutorials, vol. 13, no. 4, pp. 584-616, 2011.

[3] R. Sengupta, S. Rezaei, S. Shladover, D. Cody, S. Dickey, and H. Krishnan, "Cooperative collision warning systems: Concept definition and experimental implementation," Journal of Intelligent Transportation Systems, vol. 11, no. 3, pp. 143-155, 2007.

[4] B. van Arem, C. J. G. van Driel, and R. Visser, "The impact of cooperative adaptive cruise control on traffic-flow characteristics," IEEE Transactions on Intelligent Transportation Systems, vol. 7, no. 4, pp. 429-436, 2006.
[5] S. Shladover, C. Nowakowski, J. O'Connell, and D. Cody, "Cooperative adaptive cruise control: Driver selection of car-following gaps," in 17th ITS World Congress, 2010.

[6] S. Shladover, D. Su, and X. Lu, "Impacts of cooperative adaptive cruise control on freeway traffic flow," in 91st Transportation Research Board Annual Meeting, 2012.

[7] R. Rajamani, H. Tan, B. Law, and W. Zhang, "Demonstration of integrated longitudinal and lateral control for the operation of automated vehicles in platoons," IEEE Transactions on Control Systems Technology, vol. 8, no. 4, pp. 695-708, 2000.

[8] R. Rajamani and S. Shladover, "An experimental comparative study of autonomous and co-operative vehicle-follower control systems," Transportation Research Part C: Emerging Technologies, vol. 9, no. 1, pp. 15-31, 2001.

[9] T. Robinson, E. Chan, and E. Coelingh, "Operating platoons on public motorways: An introduction to the sartre platooning programme," in 17th World Congress on Intelligent Transport Systems, October 2010.

[10] H. Fritz, A. Gern, H. Schiemenz, and C. Bonnet, "Chauffeur assistant : a driver assistance system for commercial vehicles based on fusion of advanced acc and lane keeping," in IEEE Intelligent Vehicles Symposium, June 2004, pp. 495-500.

[11] J. Ploeg, B. T. M. Scheepers, E. van Nunen, N. van de Wouw, and H. Nijmeijer, "Design and experimental evaluation of cooperative adaptive cruise control," in Proc. 14th Int Intelligent Transportation Systems (ITSC) IEEE Conf, 2011, pp. 260-265.

[12] http://www.gcdc.net/.

[13] K. Lidström, K. Sjöberg, U. Holmberg, J. Andersson, F. Bergh, M. Bjäde, and M. Spencer, "A modular cacc system integration and design," IEEE Transactions on Intelligent Transportation Systems, vol. 13, no. 3, pp. 1050-1061, 2012.

[14] M. Nieuwenhuijze, T. van Keulen, S. Öncü, B. Bonsen, and H. Nijmeijer, "Cooperative driving with a heavy-duty truck in mixed traffic: Experimental results," IEEE Transactions on Intelligent Transportation Systems, vol. 13, no. 3, pp. 1026-1032, 2012.

[15] L. Guvenç, I. Uygan, K. Kahraman, R. Karaahmetoglu, I. Altay, M. Sentürk, M. Emirler, A. Karci, A. Guvenc, E. Altug, M. Turan, E. Bozkurt, U. Özgüner, K. Redmill, A. Kurt, and B. Efendioglu, "Cooperative adaptive cruise control implementation of team mekar at the grand cooperative driving challenge," IEEE Transactions on Intelligent Transportation Systems, vol. 13, no. 3, pp. 1062-1074, 2012.

[16] A. Geiger, M. Lauer, F. Moosmann, B. Ranft, H. Rapp, C. Stiller, and J. Ziegler, "Team annieway's entry to the 2011 grand cooperative driving challenge," IEEE Transactions on Intelligent Transportation Systems, vol. 13, no. 3, pp. 1008-1017, 2012.

[17] R. Kianfar, B. Augusto, A. Ebadighajari, U. Hakeem, J. Nilsson, A. Raza, R. Tabar, V. N., C. Englund, P. Falcone, S. Papanastasiou, L. Svensson, and H. Wymeersch, "Design and experimental validation of a cooperative driving system in the grand cooperative driving challenge," IEEE Transactions on Intelligent Transportation Systems, vol. 13, no. 3, pp. 994-1007, 2012.

[18] D. Swaroop and J. Hedrick, "String stability of interconnected systems," IEEE Transactions on Automatic Control, vol. 41, no. 3, pp. 349-357, 1996.

[19] D. Swaroop, J. Hedrick, C. Chien, and P. Ioannou, "A comparision of spacing and headway control laws for automatically controlled vehicles," Vehicle System Dynamics: International Journal of Vehicle Mechanics and Mobility, vol. 23, no. 1, pp. 597-625, 1994.

[20] M. Schönhof and D. Helbing, "Empirical features of congested traffic states and their implications for traffic modeling," Transportation Science, vol. 41, no. 2, pp. 135-166, 2007.

[21] L. Xiao and F. Gao, "Practical string stability of platoon of adaptive cruise control vehicles," IEEE Transactions on Intelligent Transportation Systems, vol. 12, no. 4, pp. 1184-1194, 2011.

[22] W. B. Dunbar and D. S. Caveney, "Distributed receding horizon control of vehicle platoons: Stability and string stability," IEEE Transactions on Automatic Control, vol. 57, no. 3, pp. 620-633, 2012.

[23] F. Bu, H.-S. Tan, and J. Huang, "Design and field testing of a cooperative adaptive cruise control system," in Proc. American Control Conf. (ACC), 2010, pp. 4616-4621.

[24] J. Nowakowski, C.and OConnell, S. Shladover, and D. Cody, "Cooperative adaptive cruise control: Driver acceptance of following gap settings less than one second," in Proceedings of the Human Factors and Ergonomics Society 54th Annual Meeting. The Human Factors and Ergonomics Society, 2010.

[25] G. Naus, R. Vugts, J. Ploeg, M. van de Molengraft, and M. Steinbuch, "String-stable cacc design and experimental validation: A frequency- 
domain approach," IEEE Transactions on Vehicular Technology, vol. 59, no. 9, pp. 4268-4279, 2010.

[26] G. Naus, R. Vugts, J. Ploeg, R. van de Molengraft, and M. Steinbuch, "Cooperative adaptive cruise control, design and experiments," in Proc. American Control Conf. (ACC), 2010, pp. 6145-6150.

[27] E. Shaw and J. K. Hedrick, "String stability analysis for heterogeneous vehicle strings," in Proc. American Control Conference, 2007, pp. 3118 3125.

[28] D. Swaroop, "String stability of interconnected systems: An application to platooning in automated highway systems," Ph.D. dissertation, UC Berkeley, 1994.

[29] G. Naus, R. Vugts, J. Ploeg, M. Van de Molengraft, and M. Steinbuch, "Towards on-the-road implementation of cooperative adaptive cruise control," in 16th World Congr. Exhib. ITS World, 2009.

[30] V. Milanes, J. Villagra, J. Perez, and C. Gonzalez, "Low-speed longitudinal controllers for mass-produced cars: A comparative study," IEEE Transactions on Industrial Electronics, vol. 59, no. 1, pp. 620-628, jan. 2012.

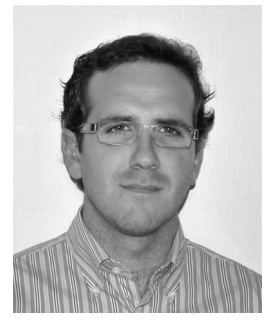

Vicente Milanés received the Ph.D. degree in electronic engineering from University of Alcala, Madrid, Spain, in 2010. He was with the AUTOPIA program at the Center for Automation and Robotics (UPM-CSIC, Spain) from 2006 to 2011. From 2012, he is a Fulbright fellow at California PATH, UC Berkeley.

Dr. Milanés has been awarded with the Best Paper Award in three conferences and his $\mathrm{PhD}$ has received two national and one international awards. His research interests include autonomous vehicles, fuzzylogic control, intelligent traffic and transport infrastructures, and vehicleinfrastructure cooperation.

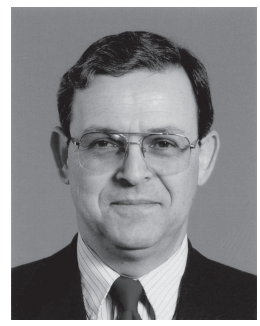

Steven Shladover is the Program Manager, Mobility at the California PATH Program of the Institute of Transportation Studies of the University of California at Berkeley. He joined the PATH Program in 1989, after eleven years at Systems Control, Inc. and Systems Control Technology, Inc., where he led the company's efforts in transportation systems engineering and computer-aided control engineering software products. Dr. Shladover received all of his degrees in mechanical engineering, with a specialization in dynamic systems and control, from M.I.T., where he began conducting research on vehicle automation in $1973 . \mathrm{He}$ has been active in ASME (former Chairman of the Dynamic Systems and Control Division), SAE (ITS Division) and the Transportation Research Board (Chairman of the Committee on Intelligent Transportation Systems from 20042010, and member of the Committee on Vehicle-Highway Automation from its founding until 2010), and was the chairman of the Advanced Vehicle Control and Safety Systems Committee of the Intelligent Transportation Society of America from its founding in 1991 until 1997. Dr. Shladover leads the U.S. delegation to ISO/TC204/WG14, which is developing international standards for "vehicle-roadway warning and control systems".

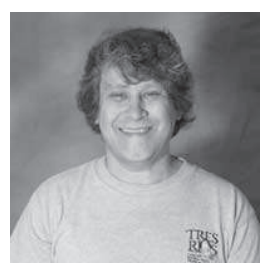

John Spring received an MS degree in Physics from San Francisco State University in 2000. He worked as a Computer Systems Engineer at Lawrence Berkeley National Laboratory from 1997 to 2006, and then as a Research and Development Engineer at PATH/UC Berkeley from 2006 to present. His specialties include controls systems and data acquisition systems engineering.

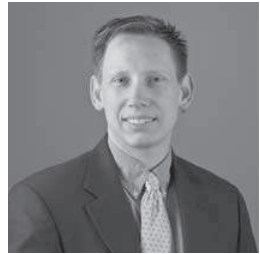

Christopher Nowakowski joined California PATH in 2002, and he is now the Senior Human Factors Engineer in the program. He has a B.S. in Civil Engineering from Bradley University and a M.S. in Industrial Engineering from the University of Michigan, where he specialized in automotive human factors engineering. He has been conducting driving behavior studies, both simulator and onthe-road experiments, since 1996 when he was a graduate student research assistant at the University of Michigan Transportation Research Institute. His research interests include the observation of driving behavior, Driver-Vehicle Interface (DVI) and Advanced Driver Assistance Systems (ADAS) design, and the field testing of Intelligent Transportation Systems (ITS) devices.

$\mathrm{He}$ is a member of the Human Factors and Ergonomics Society's Surface Transportation Group, and he is a member of the Society of Automotive Engineers' (SAE) Safety and Human Factors Steering Committee. He also currently co-chairs the SAE Safety and Human Factors Committee on Adaptive Cruise Control and Forward Collision Warning Standards.

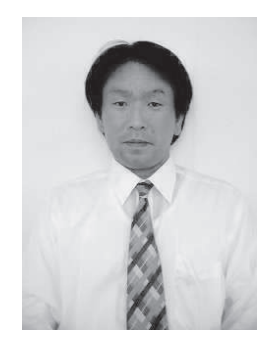

Hiroshi Kawazoe obtained the bachelor degree in electronic engineer from the Tokai University, Japan in 1987.He works for Nissan Motor Co., Ltd. His work is on the development of the advanced driver support system using communication technologies for safety and efficiency improvements. His research interests include self-controlled vehicle and infrastructure cooperation.

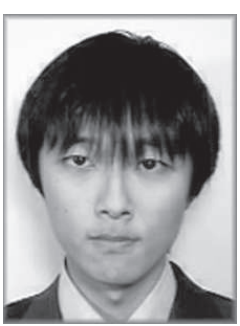

Masahide Nakamura received a M.ME. in Advanced Mechanical Science and Engineering from the Niigata University, Niigata, Japan, in 2002. He works for Nissan Motor Co., Ltd. His work is on the development of the advanced driver support system using communication technologies for safety and efficiency improvements. His research interests include self-controlled vehicle and infrastructure cooperation. 